\title{
Modeling of filtration of 2-types particles suspension in a porous medium
}

\author{
Yuri Galaguz ${ }^{1}$, Galina Safina, ${ }^{1, *}$ \\ ${ }^{1}$ Moscow state university of civil engineering, Yaroslavskoye shosse, 26, Moscow, Russia, 129337
}

\begin{abstract}
The filtration problem describes the process of concreting loose soil with a liquefied concrete solution. The filtration of 2-types particles suspension in a homogeneous porous medium with a size-exclusion particles retention mechanism is considered. The difference in the filtration coefficients of 2-types particles leads to the separation of the filtration domain into two zones, in one of which two types of particles are deposited and in another - only particles of one type are deposited. In this paper, the mobile boundary of two zones is calculated, and numerical solution of the problem is calculated.
\end{abstract}

\section{Introduction}

The calculation of filtration problem is important for concreting loose soil. Liquefied concrete penetrates into the porous soil and, solidifying, strengthens it. Due to the filtration of solid suspended particles in porous filters, primary purification of liquid industrial wastes and municipal wastewater occurs [1-3].

The classical model of filtration of a suspension in a porous medium considers the process of transport and deposition of identical particles moving with a constant velocity [4, 5]. The mathematical model includes the equation of mass transfer and the kinetic equation that determines the growth of the deposit in proportion to the concentration of suspended particles. The coefficient of proportionality in the kinetic equation is called the filtration coefficient. Modern models take into account the dependence of the porosity and permeability of a porous medium on the deposit size and various mechanisms of particle capture [6-9].

A mechanical model of particles retention, based on a comparison of particle and pore sizes is considered in this paper. It is assumed that the particles freely pass through large pores and get stuck at the entrance of small pores, the dimensions of which are smaller than the particle diameter [10]. In contrast to the classical model, a suspension containing particles of two different types is considered. Particles that differ in size, shape, or density move in a porous medium with the same velocities. The concentrations of suspended and retained particles satisfy kinetic equations with different filtration coefficients. The condition of non-negativity of the filtration coefficients leads to the separation of the filtration domain into two zones. At small times, in the first zone both types of particles are retained. When one of the filter coefficients turns to zero, the corresponding suspended

\footnotetext{
${ }^{*}$ Corresponding author: minkinag@mail.ru
} 
particles begin to flow freely through the porous medium. In this zone only the particles of the second type are retained.

For some filtration models, the exact solutions are obtained or the asymptotic formulas are constructed [11-17]. In the general case, the analytical solution is absence and numerical calculations are carried out $[18,19]$. In this paper, the mobile boundary of two zones is computed numerically and numerical solution of the problem is obtained.

\section{The problem formulation}

In the domain $\Omega=\{0<x<1, t>0\}$ consider a first-order quasilinear hyperbolic system with respect to the concentrations of suspended and retained particles of two types $C_{i}(x, t) ; S_{i}(x, t) ; i=1,2$

$$
\begin{aligned}
& \frac{\partial C_{i}}{\partial t}+\frac{\partial C_{i}}{\partial x}+\frac{\partial S_{i}}{\partial t}=0 ; \\
& \frac{\partial S_{i}}{\partial t}=\Lambda_{i}(S) C_{i} ; i=1,2
\end{aligned}
$$

with the boundary condition

$$
x=0: C_{i}=p_{i}
$$

and the initial conditions

$$
t=0: C_{i}=0, S_{i}=0 .
$$

Here $S=S_{1}+S_{2}$ is the total deposit concentration; the filtration coefficients are $\Lambda_{i}(S) \geq 0, i=1,2$.

At the initial time the porous medium does not contain any particles (condition 3 ). At $t=0$ a suspension with constant suspended particles concentrations $p_{i}$ is injected at the filter inlet (condition 4). The discontinuity of the boundary conditions (3) and (4) at the origin extends over the characteristic $t=x$ of the hyperbolic system (1)-(4). This characteristic is the concentrations front of the suspended and retained particles, moving with a constant velocity $v=1$. The concentration front divides the domain $\Omega$ into two subdomains $\Omega_{0}=\{0<x<1,0<t<x\}$ and $\Omega_{S}=\{0<x<1, t>x\}$. In the subdomain $\Omega_{0}$ the system (1)-(4) has a zero solution, in the subdomain $\Omega_{S}$ the solution is positive.

Assume that the filtration coefficient $\Lambda_{1}(S)$ turns to zero at a point $S=S_{0}$, and the coefficient $\Lambda_{2}(S)$ is positive at this point:

$$
\Lambda_{1}(S)>0,0 \leq S<S_{0} ; \quad \Lambda_{1}(S)=0, S \geq S_{0} ; \quad \Lambda_{2}(S)>0,0 \leq S \leq S_{0} .
$$

When $S<S_{0}$ particles of two sizes are retained in a porous medium. When $S>S_{0}$ the filtration coefficient $\Lambda_{1}(S)=0$ and particles of the first type pass freely through the porous medium. In this zone only particles of the second type are retained. The mobile boundary of the two filtration zones is given by the equation

$$
\Lambda_{1}(S(x, t))=0 \text {. }
$$




\section{Numerical modeling}

A numerical solution of the problem is obtained by the method of finite differences using an explicit difference scheme. To fulfill the convergence conditions and the adequacy of the approximate solution on the discontinuity line $t=x$ steps in time and coordinate are chosen the same $\tau=h=0,001$ [20].

A numerical calculation is performed for a 2-types particles suspension in the case of linear filtration coefficients

$$
\Lambda_{i}(S)=\lambda_{i}-a_{i} S ; i=1,2
$$

for initial conditions $p_{1}=1, p_{2}=1$.

The parameters of the functions (6) are chosen as follows $\lambda_{1}=1, \lambda_{2}=2, a_{1}=1, a_{2}=1$.

The concentrations front of suspended and retained particles and the mobile boundary of the two filtration zones are shown in Fig. 1. In the case of linear filtration coefficients (6) the boundary line is close to a straight line.

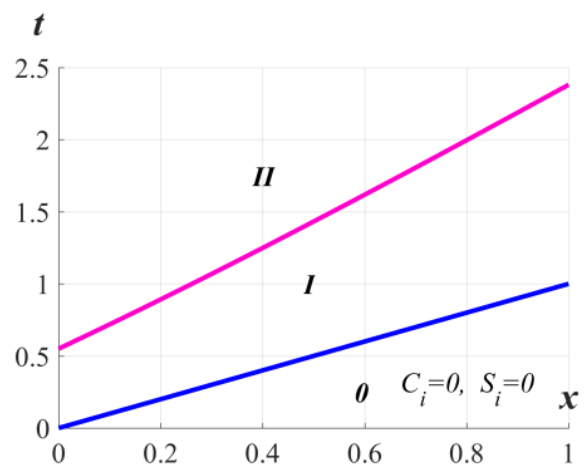

Fig. 1. The concentrations front and the mobile boundary of filtration zones.

In zone 0 (the domain $\Omega_{0}$ ) there are no particles and the solution is zero. In zone I the particles of two sizes are retained. In zone II the particles of type 1 pass only through large pores and do not get stuck, only particles of type 2 are retained.

Below 3-dimensional graphs of the suspended particles concentrations $C_{1}, C_{2}$ (Fig. 2, a), b)), the retained particles concentrations $S_{1}, S_{2}$ (Fig.3 a), b)) and the total deposit concentration $S$ (Fig. 4) are shown.

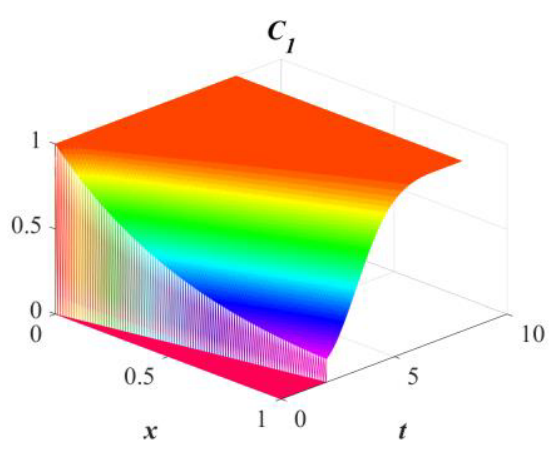

Fig. 2. a) Suspended particles $C_{1}(x, t)$.

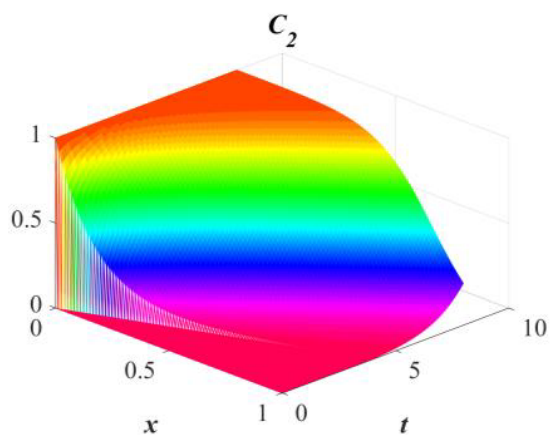

b) Suspended particles $C_{2}(x, t)$. 


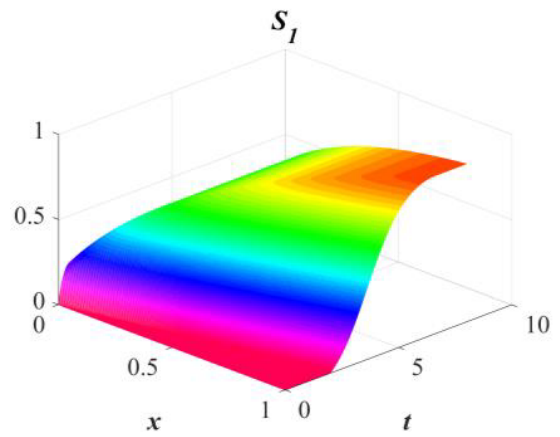

Fig. 3. a) Retained particles $S_{1}(x, t)$.

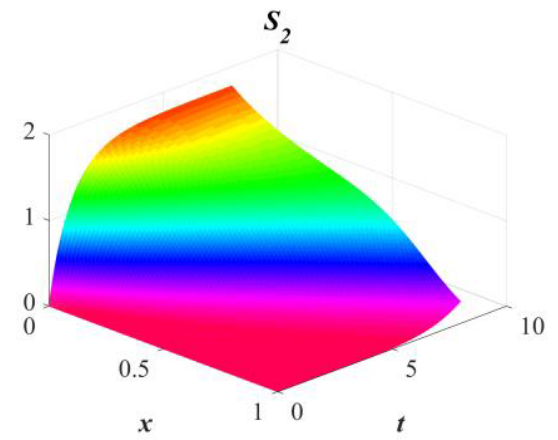

b) Retained particles $S_{2}(x, t)$.

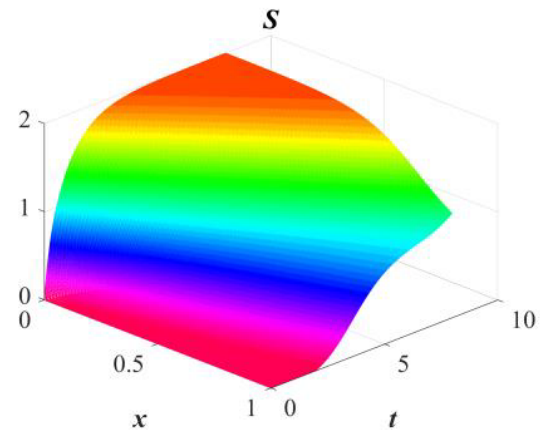

Fig. 4. Total retained particles concentration $S(x, t)$.

Fig. 2 shows that the solution $C_{i}(x, t), i=1,2$ experiences a jump at the concentration front $t=x$; on this line the solution $S_{i}(x, t), i=1,2$ and the total retained particles concentration $S(x, t)$ are continuous and have a break (Fig. 3, 4).

On the boundary (5) of two filtration zones the solution is smooth (Fig. 2-4). On this line the solution undergoes a weak discontinuity: first-order derivatives $\partial S_{i} / \partial t, i=1,2$ have a break and second-order derivatives are discontinuous (Fig. 5).

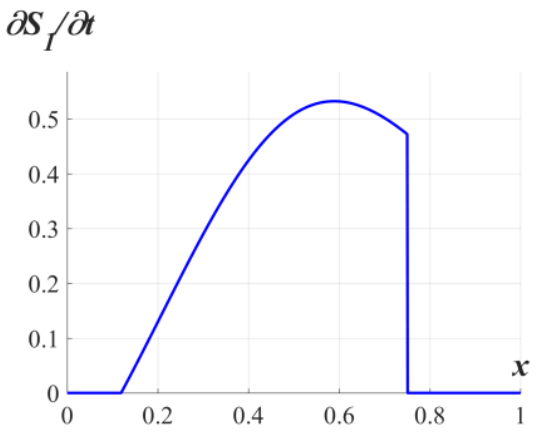

Fig. 5. The derivative $\partial S_{1} /\left.\partial t\right|_{t=0.75}$.

The left zero part of the graph corresponds to zone II, in which the particles of the first type do not deposited. The part of the positive derivative corresponds to zone I, in which all 
types of particles are filtered. At the point $x=0.75$ the graph reaches the front of the concentrations, there are no particles before of the concentration front on the segment $0.75 \leq x \leq 1$ and the derivative is zero there.

In Fig. 6, 7 the graphs of suspended and retained particles concentrations at fixed time $t=1.5$ are presented.

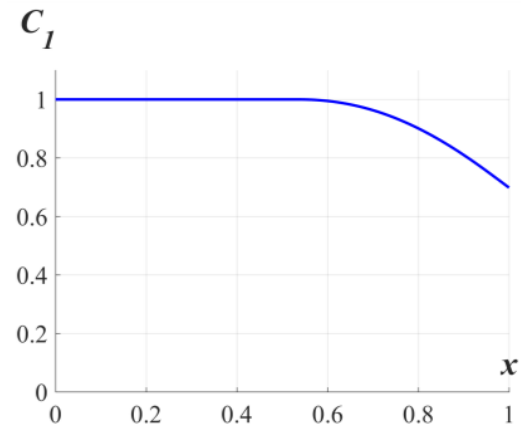

Fig. 6. a) $\left.C_{1}(x, t)\right|_{t=1.5}$.

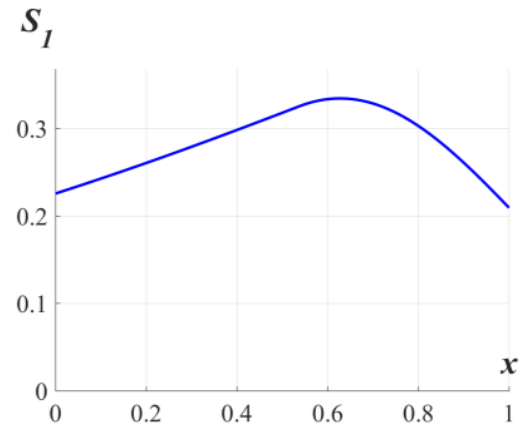

Fig. 7. a) $\left.S_{1}(x, t)\right|_{t=1.5}$.

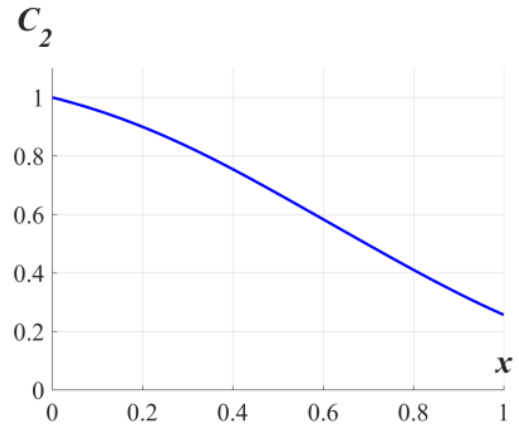

b) $\left.C_{2}(x, t)\right|_{t=1.5}$.

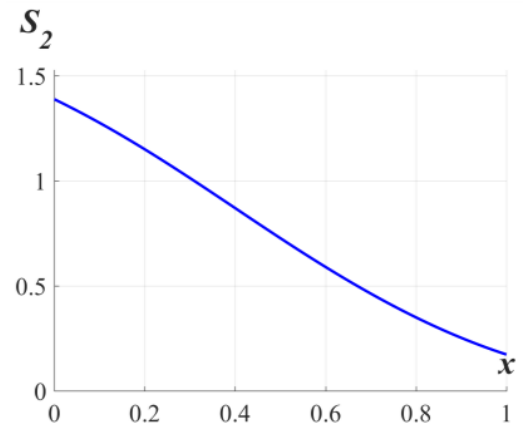

b) $\left.S_{2}(x, t)\right|_{t=1.5}$.

The concentrations of suspended particles $\left.C_{i}(x, t)\right|_{t=1.5}, i=1,2$ decrease as the distance $x$ increases to the filter inlet (Fig. 4). At the filter inlet $x=0$ the filtration of particles of the first type terminates at the time $t=t_{0}$ specified by the equation $\Lambda_{1}\left(S\left(0, t_{0}\right)\right)=0$, and at the filter outlet at the time $t=t_{1}$, where $\Lambda_{1}\left(S\left(1, t_{1}\right)\right)=0$. For a fixed $t<t_{0}$ the concentration $C_{1}(x, t)<1$ at $0<x<1$. At $t_{0}<t<t_{1}$ the concentration $C_{1}(x, t)=1$ at $S(x, t)<S_{0}$ and $C_{1}(x, t)<1$ at $S(x, t)>S_{0}$. At $t>t_{1}$ all over the porous medium only particles of the second type are retained and $C_{1}(x, t)=1 ; 0<x<1$.

The retained particles concentration $\left.S_{2}(x, t)\right|_{t=1.5}$ decreases as the distance $x$ increases to the filter inlet (Fig. 7 b)). At a fixed $t<t_{0}$ the concentrations $S_{i}(x, t) ; i=1,2$ and $S(x, t)$ decrease for all $0<x<1$. When $t_{0}<t<t_{1}$ the concentration $S_{1}(x, t)$ reaches the limiting constant value $S_{1}^{0}<S_{0}$ on a interval $0 \leq x \leq x_{0}(t) ; x_{0}(t)<1$, where $S\left(x_{0}(t), t\right)=S_{0}$ (Fig. 7 a)). When $x_{0}(t)<x<1$ we have $S(x, t)<S_{0}$ and the function $S_{1}(x, t)$ decreases. At $t>t_{1}$ all over the filter the retained particles concentration of the type 1 is constant: $S_{1}(x, t)=S_{1}^{0}$. 
In Fig. 8, 9 the graphs of concentrations of suspended and retained particles at a fixed distance from the filter input $x=0.5$ are shown.

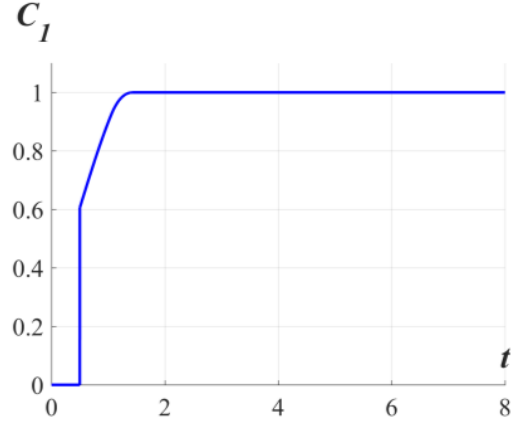

Fig. 8. a) $\left.C_{1}(x, t)\right|_{x=0.5}$.

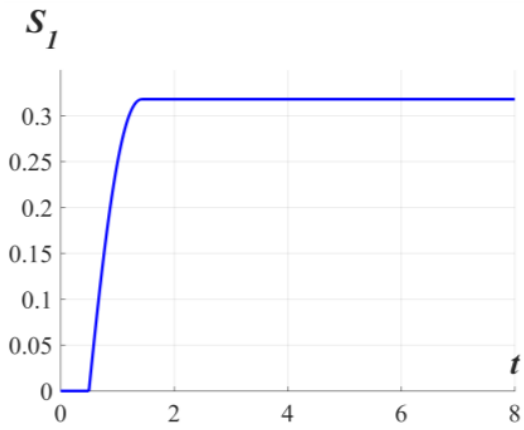

Fig. 9. a) $\left.S_{1}(x, t)\right|_{x=0.5}$.

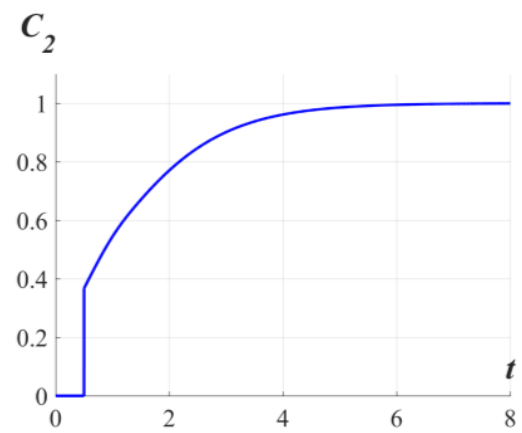

b) $\left.C_{2}(x, t)\right|_{x=0.5}$.

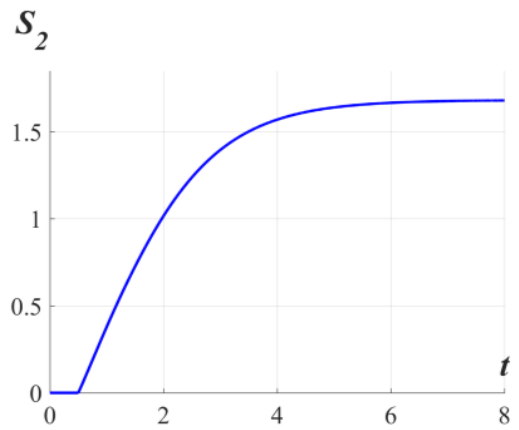

b) $\left.S_{2}(x, t)\right|_{x=0.5}$.

The concentrations of suspended and retained particles are zero before the concentration front (Fig. 8, 9). At the front of concentration $t=x$, the concentrations of suspended particles $C_{i}(x, t) ; i=1,2$ are discontinuous, and the concentrations of retained particles $S_{i}(x, t) ; i=1,2$ are continuous and fractured. On the boundary line of the two filtration zones the solution does not lose its smoothness.

For a fixed $x$ all concentrations are non-decreasing. When the total deposit reaches the value $S_{0}$ the concentrations of the suspended and retained particles of the first type become constant: $S_{1}(x, t)=S_{1}^{0}, C_{1}(x, t)=1$. The concentrations of suspended and retained particles of the second type $S_{2}(x, t), C_{2}(x, t)$ constantly increase and tend to limit values.

\section{Conclusion}

A numerical solution of the problem of filtration of a suspension with solid particles of two types in a porous medium for linear and linearly-constant filtration coefficients is constructed. The filtering domain is divided into two zones. In one zone particles of two types are deposited. And in the other - only one type. A mobile border of two zones is constructed; the graphs of concentrations of suspended and retained particles of two types are presented. It is shown that the solution at the boundary of two filtration zones is smooth. 


\section{References}

1 P.G. Bedrikovetsky, Mathematical Theory of Oil and Gas Recovery (Kluwer 1994)

2 S.A. Bradford, S. Torkzaban, Vad. Zone J. 7 (2008)

3 C. Noubactep, S. Care, Chem. Eng. J. 163 (2010)

4 J. P. Herzig, D. M. Leclerc, P. Legoff, Ind. Eng. Chem. 62 (1970)

5 E.A. Vyazmina, P.G. Bedrikovetskii, A.D. Polyanin, Theor. Found. Chem. Eng. 41, 5 (2007)

6 Z. You, P. Bedrikovetsky, L. Kuzmina, Abstr. Appl. Anal. 2013, ID 680693 (2013)

7 H. Yuan, G. Sin, Chem. Eng. J. 168 (2011)

8 A. Santos, P. Bedrikovetsky, Transp. Porous Media 62 (2006)

9 H. Yuan, Z. You, A.A. Shapiro, P. Bedrikovetsky, Chem. Eng. J. 226 (2013)

10 A. Badalyan, Z. You, K. Aji, P. Bedrikovetsky, T. Carageorgos, A. Zeinijahromi, Rev. Sci. Instr. 85 (2014) art. No.: 015111

11 L.I. Kuzmina, Yu.V. Osipov, Int. J. Comp. Civ. Str. Eng. 12, 32016

12 L.I. Kuzmina, Yu.V. Osipov, Matec Web Conf. 86, 01005 (2016)

13 S. Borazjani, A. J. Roberts, P. Bedrikovetsky, Appl. Math. Lett. 53 (2015)

14 M. Hayek, G. Kozakovski, S. Churakov, Wat. Res. Res. 47 (2011)

15 Z. You, Y. Osipov, P. Bedrikovetsky, L. Kuzmina, Chem. Eng. J. 258 (2014)

16 L.I. Kuzmina, Yu.V. Osipov, Procedia Eng. 111 (2015)

17 L.I. Kuzmina, Yu.V. Osipov, Vestnik MGSU, 2 (2016)

18 Yu.P. Galaguz, G.L. Safina, Procedia Eng. 153 (2016)

19 Y. Galaguz, G. Safina, Matec Web Conf. 86, 03003 (2016)

20 E.F. Toro, Riemann solvers and numerical methods for fluid dynamics, (Springer, Dordrecht, 2009) 